\title{
Ethnopharmacological survey: a selection strategy to identify medicinal plants for a local phytotherapy program
}

\author{
Flávia Liparini Pereira1, José Martins Fernandes², João Paulo Viana Leite ${ }^{1, *}$
}

${ }^{1}$ Center for Biological and Health Sciences, Department of Biochemistry and Molecular Biology, Federal University of Viçosa, ${ }^{2}$ Department of Plant Biology, Federal University of Viçosa

\begin{abstract}
Ethnopharmacological studies are important for documenting and protecting cultural and traditional knowledge associated with the medical use of biodiversity. In this paper, we present a survey on medicinal plants used by locals in a community of Nova Viçosa, Viçosa, MG, Brazil, as a strategy to select medicinal plants for a phytotherapy-based local healthcare program. Eleven knowledgeable local informants were chosen by snowball sampling and interviewed about the use of medicinal plants. Plant samples were collected, herborised and then identified using traditional techniques and specialised literature. We sampled 107 medicinal plant species belonging to 86 genera and 39 families, predominantly Asteraceae with 16 species. Costus spicatus (Jacq.) Sw, M. pulegium L., Rosmarinus officinalis L. and Ruta graveolens L. were found to have Consensus of Main Use corrected (CMUc) values above 50\%, which were in agreement with the traditional uses described by the informants. However, species with CMUc values equal to or above $20 \%$, combined with the scientific information survey, were also used to select medicinal plants for the phytotherapy-based local healthcare program. The selection of medicinal plants based on the CMUc index from this particular community, in combination with the scientific survey, appears to be an effective strategy for the implementation of phytotherapy programs.
\end{abstract}

Uniterms: Ethnopharmacology. Medicinal plants. Phytotherapy.

Estudos etnofarmacológicos são importantes no registro e na preservação de conhecimentos de uma cultura tradicional associada ao uso medicinal da biodiversidade. No presente trabalho, foi realizado o levantamento das plantas medicinais utilizadas por conhecedores populares na comunidade de Nova Viçosa, Viçosa, Minas Gerais, Brasil, como ferramenta para auxiliar na seleção de espécies vegetais visando à implantação de um programa de fitoterapia local na comunidade estudada. Participaram 11 conhecedores escolhidos por amostragem Bola de Neve e submetidos a entrevistas semiestruturadas. Amostras dos espécimes foram coletadas, herborizadas e identificadas conforme metodologias usuais e literatura especializada. Foram amostradas 107 espécies medicinais pertencentes a 86 gêneros e 39 famílias botânicas, destacando-se Asteraceae com 16 espécies. Costus spicatus (Jacq.) Sw., Mentha pulegium L., Rosmarinus officinalis L. e Ruta graveolens L. apresentaram Consenso de Uso Principal corrigido (CUPc) superior a 50\%, evidenciando consensos de uso popular entre os entrevistados. Espécies com CUPc igual ou superior a $20 \%$, juntamente com o levantamento de informações em bases científicas, foram utilizadas para selecionar as espécies para o programa de fitoterapia local. A seleção de plantas medicinais, levando-se em consideração o índice de CUPc obtido de uma determinada comunidade aliado ao levantamento científico, mostra-se como estratégia a ser considerada na implantação de programas fitoterápicos.

Unitermos: Etnofarmacologia. Plantas medicinais. Fitoterapia.

\footnotetext{
*Correspondence: J. P. V. Leite. Departamento de Bioquímica e Biologia Molecular, Universidade Federal de Viçosa. Av. P.H.Rolfs, s/n. Campus Universitário, 36570-000 - Viçosa - MG, Brasil. Tel. +55 313899 3044; Fax: +55 313899 2373. E-mail: jpvleite@ufv.br
} 


\section{INTRODUCTION}

Ethnopharmacology is defined as the interdisciplinary scientific exploration of the biologically active agents of plants, animals and other substances traditionally employed or observed by man (Bruhn, Holmstedt, 1981; ISE, 2012). Furthermore, it combines information from traditional users of medicinal flora with chemical and pharmacological studies, allowing for the formulation of hypotheses about the pharmacological activities and substances responsible for the reported therapeutic activities (Elisabetsky, 2003).

Data from the World Health Organization (WHO) show that approximately $80 \%$ of the world population depends on medicinal plants or herbal medicines for primary health care. Developing countries hold $67 \%$ of plant biodiversity on the planet, highlighting their participation in the process of enhancing the use of medicinal plants in health care promoted by the WHO (Brasil, 2006). However, there has been a major loss of traditional knowledge regarding medicinal plants in these countries, and the degradation of natural environments, especially tropical forests, has reduced the availability of medicinal plants (Bermúdez, Oliveira-Miranda, Velázquez, 2005).

The acculturation of modern societies, early industrialisation and the subsequent urbanisation and cultural globalisation have put traditional plant knowledge on hold in several regions (Camejo-Rodrigues, 2001, Santos et al., 2007).

In Brazil, the Ministry of Health has recently launched two important programs regarding the uses and research of medicinal plants: the National Program on Medicinal Plants and Herbal Medicines (PNPMF) (Brasil, 2009) and the National Policy on Integrative and Complementary Practices (PNPIC) (Brasil, 2006). PNPMF shares common goals aimed to ensure the safe access and rational use of medicinal plants and herbal medicines in Brazil, the development of technologies and innovations, the sustainable use of Brazilian biodiversity, and the strengthening of the productive chain of herbal products. PNPIC includes policies, actions and government responsibilities for the insertion of therapeutic practices, including phytotherapy, in the National Health System (SUS). These government initiatives have encouraged many Brazilian cities to implement phytotherapy-based healthcare programs, increasing the choice of medical treatments provided by SUS.

The state of Minas Gerais has the largest number of municipalities in the country, with 853 cities. Approximately $80 \%$ of these cities have less than 20,000 inhabitants and are known for their strong agricultural traditions (FJP, 2003). Moreover, this state comprises two major biomes, the Cerrado and Atlantic Forest, both of which are considered hotspots with high biodiversity, high endemism and severe environmental degradation (Myers et al., 2000).

In the past, the Atlantic Forest covered approximately 1 million $\mathrm{km}^{2}$, corresponding to $12 \%$ of the Brazilian territory, but today, only an estimated $5 \%$ of the original area is still intact and highly fragmented (Borém, Oliveira-Filho, 2002). Brazil is noted for having the greatest biodiversity on the planet. For instance, Brazilian ecosystems comprise approximately $22 \%$ of all of the biological species in the world. The Atlantic Forest, even with its dramatic decline, supports approximately 20,000 species of vascular plants, of which 8,000 are endemic (Marques, 2000; Mittermeier et al., 2003; Tabarelli et al., 2005). However, Brazil also has a wide range of ethnic and cultural diversity, with many traditional indigenous people and urban dwellers that cultivate medicinal plants in old home gardens (Diegues et al., 2000; Brasil, 2006; Alves et al., 2008).

An old home garden is commonly a plot of land around a house with easy access, in which residents grow or maintain many species of plants that account for part of the nutritional needs of the family, while other products are used as firewood and medicinal plants (Brito, Coelho, 2000). Home gardens are one of the oldest forms of land management. They are vital for the sustainability of people and knowledge and the management of natural environments, and they contribute to the livelihoods of certain populations (Moura, Andrade, 2007; Amaral, GuarimNeto, 2008).

This study is part of a larger project on the implementation of phytotherapy in the Health System for the state of Minas Gerais (Brasil, 2009). This is the first study that combines quantitative research on ethnopharmacology with a database of scientific information, which will help select plants for the production of phytotherapies to be provided in the public primary healthcare system. Furthermore, this study is aimed to perform a survey of medicinal plants used by the locals in the community of Nova Viçosa, Viçosa, Minas Gerais, Brazil by using the index of Consensus of Main Use corrected (CMUc), which can be implemented by local phytotherapy programs.

\section{MATERIAL AND METHODS}

\section{STUDY AREA}

The city of Viçosa is located in the southeast Brazilian "Zona da Mata" Region in the State of Minas Gerais $\left(20^{\circ} 45^{\prime} \mathrm{S}\right.$ and $\left.42^{\circ} 55^{\prime} \mathrm{W}\right)$ (Figure 1). The municipality 


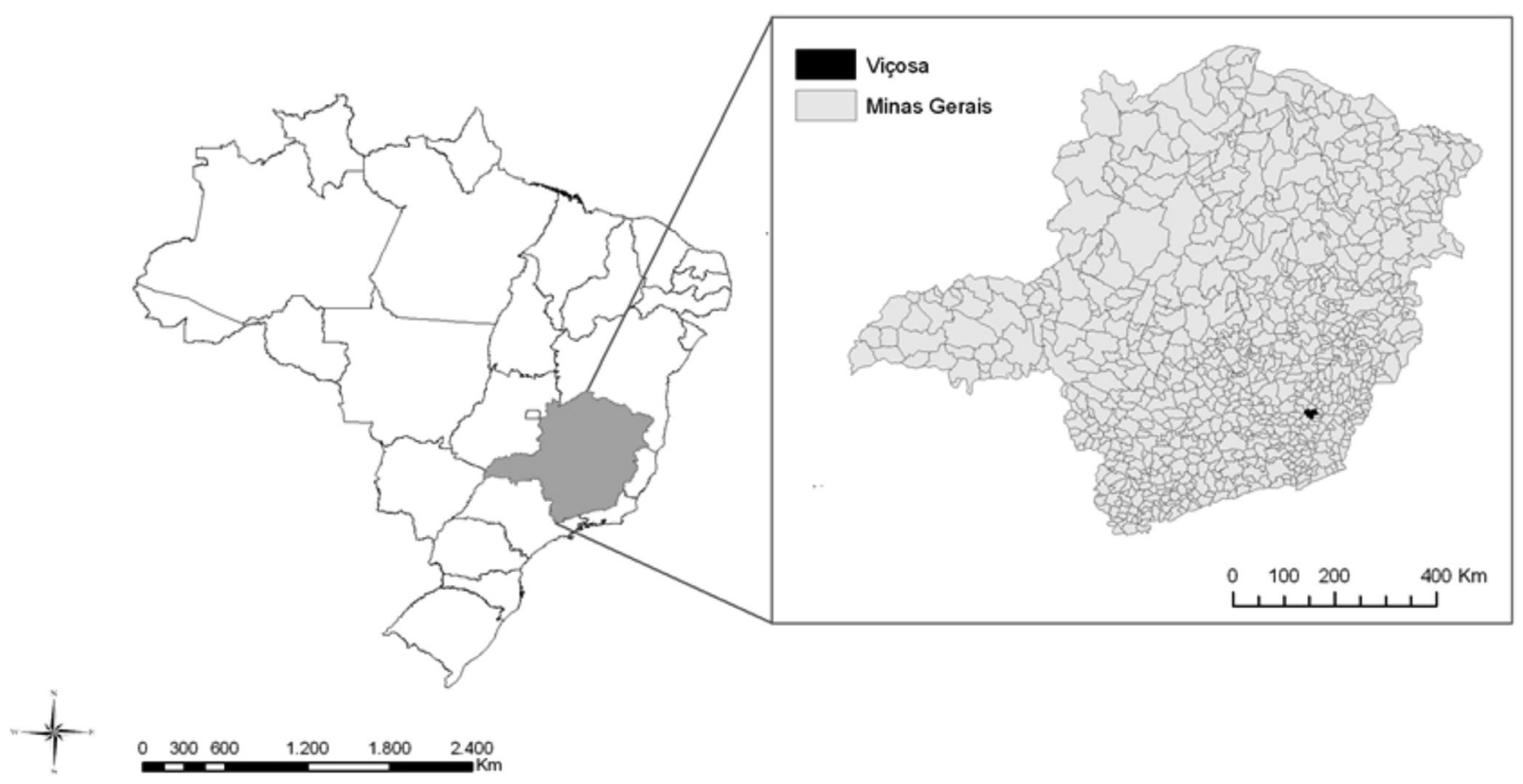

FIGURE 1 - Location of the city of Viçosa, Minas Gerais, Brazil.

comprises an area of $299 \mathrm{~km}^{2}$ and currently has approximately 72,000 inhabitants (IBGE, 2010). It was founded in the late eighteenth century, during the gold cycle, when settlers came in search of fertile land for agriculture and livestock because of the depletion of mineral deposits and the growing food shortages in the neighbouring towns (Mello, 2002).

After the gold cycle, coffee farming began to expand rapidly throughout the region. Coffee is a predatory crop based on the deforestation it caused, and coffee monoculture quickly became unproductive because of the soil (predominantly Red-Yellow Dystrophic Latosol) and relief (hilly landscape) characteristics. Plantations were replaced with new coffee plants or abandoned, and some of these areas were used for extensive cattle raising, which further hampered the natural regeneration of forests (Paula et al., 2002).

The climate in the region is mesothermal $(\mathrm{Cwb})$, with hot and rainy summers and cold and dry winters, according to the Köppen system (Arato, Martins, Ferrari, 2003). The vegetation is classified as a Montane Semideciduous Forest, a mix of deciduous and evergreen species established above a $500 \mathrm{~m}$ altitude in the Atlantic Forest domain (Veloso, Rangel-Filho, Lima, 1991).

This study was conducted in the small community of Nova Viçosa belonging to the municipality of Viçosa, MG, with a population of 8,000 inhabitants. Carvalho et al. (2004) listed several problems that residents of this district face, including precarious housing conditions, poor hygiene practices, the abandonment of a large open space that could be used as a recreational area for the benefit of the residents, and the lack of landscape and urban planning. The community also lacks the financial support to perform proper economic activities in the neighbourhood and harness the potential of public spaces to generate socio-cultural and economic benefits in the local community. The authors reported that, since 2003, a number of urban agriculture projects have been performed in the community. The aims of these programs are to improve the quality of life and food security of people occupying idle plots and backyards with vegetable and medicinal plant gardens.

\section{ETHNOPHARMACOLOGICAL SURVEY}

Eleven knowledgeable informants were interviewed about the use of medicinal plants between March and August 2008. Participant observations were also recorded during the study period in the community. Informants were selected for their recognition in the community as being knowledgeable of the medicinal properties of plants grown in their backyards. The snowball sampling method was used according to Albuquerque and Lucena (2004). All interviews were semi-structured and conducted in the presence of a local healthcare agent. The questions addressed to the knowledgeable informants dealt with information on the following: the source of traditional knowledge, the species cultivated in the backyards and used by the family, 
common names of plants, therapeutic indications, the plant parts used, and preparation methods.

During the interviews, we collected fertile and/ or sterile specimens that were subsequently herborised using traditional techniques (Bridson, Forman, 1999). The species were then identified by specialised literature and comparison with specimens at the VIC Herbarium, Federal University of Viçosa.

The species names were placed in their respective families following APG III (2009), except for Leguminosae, which was placed according to Lewis et al. (2005), and the classification has been confirmed by The International Plant Names Index (IPNI, 2009). The names of the authors are in accordance with Brummit and Powell (1992).

Data were analysed qualitatively using reports obtained through interviews. Quantitative analysis was performed by applying the index adapted by Amorozo and Gély (1988) to assess the relative importance of the medicinal plant species among the knowledgeable informants. This same index was also used to assess the consensus for the plant uses of each species mentioned by four or more informants. In this study, we considered a percentage of Consensus of Main Use corrected (CMUc) above $20 \%$ as significant.

Values for the Consensus of Main Use (CMU) and the Correction Factor (CF) of each species were applied to the formula $C M U c=C M U x C F$ to find the Consensus of Main Use corrected (CMUc) percentages.

\section{Ethnopharmacological data systematisation}

Ethnopharmacological data were compared with available scientific information on the efficacy and toxicology of the medicinal plants used from specialized databases, such as PubMed and Science Direct. We also searched national and international specialised literature officially recognised as parameters to evaluate the therapeutic efficacy and safety of the herbal drugs (Brasil, 2010). Species showing a CMUc above $20 \%$ and the scientific basis for therapeutic indications according to the informants were selected to compile a list of medicinal plants, systematised by therapeutic categories, to be included in the phytotherapy programs of the primary healthcare system.

\section{RESULTS AND DISCUSSION}

\section{Profiles of the Informants}

Ninety-one per cent of interviewees were women, and $82 \%$ were over 50 years of age. The average age of the informants was 63.1 years (with a range of 37-83 years). Most informants (73\%) acquired knowledge about medicinal plants from their families, which was associated or not with other forms of knowledge acquired by personal experiences, neighbours, books, television and teachers. We also found that the majority of informants $(91 \%)$ have passed on their knowledge whenever they have been asked. However, many interviewees reported their children and grandchildren lack interest in this knowledge. This scenario characterises a risk of loss for an oral tradition, emphasising the importance of documenting traditional knowledge about plant use. It is also important to note that this knowledge is a vital strategy in drug discovery and in the valuation of biodiversity and is a major part of the patrimony of a nation (Brasil, 2012).

\section{Ethnopharmacological study}

Knowledgeable informants of the community of Nova Viçosa reported the use of 107 medicinal plant species, belonging to 86 genera and 39 families (Table I). Cultivation of these medicinal species was recorded in all of the sampled yards, with an average of 23 (a minimum of 11 and a maximum of 49) species in each yard, totalling 258 plant species used in traditional medicine.

The most frequently mentioned plant families in this study, with the largest number of species, were Lamiaceae with 17 species (mentioned 64 times) and Asteraceae with 16 species (mentioned 36 times). Asteraceae, which contains 1,535 genera and 32,000 species and is considered the largest family of angiosperms (Pruski, Sancho, 2004), and Lamiaceae, which contains 300 genera and 7,500 species (Souza, Lorenzi, 2009), including a considerable number of aromatic species, are among the families with the greatest diversity of species mentioned in studies of medicinal plants.

Similar results were found in studies from different regions of the country. Pilla, Amorozo and Furlan (2006), in a study conducted in the district of Martim Francisco, a municipality of Mogi Mirim, São Paulo, mentioned the use of 107 species. Among these species, 14 were Lamiaceae and 12 were Asteraceae. Ming and Junior (2005), in a study conducted in the Extractive Reserve of Chico Mendes in the State of Acre; Pinto, Amorozo and Furlan (2006), in the rural communities of the Atlantic Forest domain in Itacaré (BA); and Santos, Lima and Ferreira (2008), in Ariquemes, the State of Rondônia, also found similar data on the number of species and the most representative botanical families.

The predominant habits of the surveyed species were herbaceous $(60 \%)$, subshrub (15\%), shrub (14\%), tree 
TABLE I - Medicinal species used by locals in the community of Nova Viçosa, Viçosa, Minas Gerais, Brazil

\begin{tabular}{|c|c|c|c|c|c|c|c|}
\hline Family/Species & Common name & $\begin{array}{l}\text { Used } \\
\text { part }\end{array}$ & $\begin{array}{l}\text { Method of } \\
\text { preparation }\end{array}$ & Mentioned use & Habit & $\begin{array}{l}\text { Status } \\
\text { of origin } \\
\text { in South } \\
\text { America* }\end{array}$ & $\begin{array}{c}\text { Number of times } \\
\text { mentioned by } \\
\text { informants }\end{array}$ \\
\hline $\begin{array}{l}\text { Sambucus australis Cham. \& } \\
\text { Schltdl. }\end{array}$ & Sabugueiro & If & Infusion & Influenza; cough & $\mathrm{sb}$ & $\mathrm{na}^{1}$ & 1 \\
\hline \multicolumn{8}{|l|}{ AMARANTHACEAE } \\
\hline Amaranthus sp & Caruru & lf & Fresh & Anaemia & he & $\mathrm{na}^{4}$ & 1 \\
\hline Chenopodium ambrosioides L. & $\begin{array}{l}\text { Santa maria; erva de } \\
\text { santa maria }\end{array}$ & lf; fl & Infusion & $\begin{array}{l}\text { Abdominal pain; } \\
\text { vermifuge; fleas }\end{array}$ & he & $\mathrm{na}^{1}$ & 3 \\
\hline Iresine herbstii Hook. & Sangue de cristo & lf & Infusion & Suspension & $\mathrm{sb}$ & $\mathrm{na}^{2}$ & 1 \\
\hline Allium fistulosum L. & Cebolinha verde & If & Fresh & $\begin{array}{c}\text { Appetite } \\
\text { stimulator }\end{array}$ & he & $e x^{1}$ & 1 \\
\hline \multicolumn{8}{|l|}{ ANACARDIACEAE } \\
\hline Mangifera indica $\mathrm{L}$. & Manga & If & Infusion & Influenza & $\mathrm{cl}$ & $\mathrm{ex}^{3}$ & 1 \\
\hline Schinus terebinthifolia Raddi & Aroeira & lf; ba & $\begin{array}{l}\text { Infusion } \\
\text { (topical) }\end{array}$ & $\begin{array}{l}\text { Allergy; itching; } \\
\text { wounds }\end{array}$ & $\mathrm{cl}$ & $\mathrm{na}^{1}$ & 1 \\
\hline \multicolumn{8}{|l|}{$\overline{\text { APIACEAE }}$} \\
\hline Apium graveolens $\mathrm{L}$. & Aipo & lf; bra & Infusion & $\begin{array}{l}\text { Blood pressure; } \\
\text { gas; belching; } \\
\text { abdominal pain }\end{array}$ & he & $e x^{1}$ & 3 \\
\hline Eryngium foetidum $\mathrm{L}$. & $\begin{array}{l}\text { Coentro do chile; } \\
\text { coentro }\end{array}$ & If & Infusion & $\begin{array}{c}\text { Digestive } \\
\text { problem; pre- } \\
\text { menstrual tension }\end{array}$ & he & $n a^{1}$ & 2 \\
\hline \multicolumn{8}{|l|}{ ARACEAE } \\
\hline Colocasia esculenta (L.) Schott. & Inhame & tu & Decoction & $\begin{array}{l}\text { Chilblains; } \\
\text { detoxifying; } \\
\text { depurative }\end{array}$ & he & $e^{2}$ & 1 \\
\hline \multicolumn{8}{|l|}{ ASPARAGACEAE } \\
\hline Agave sp & Piteira & If & $\begin{array}{l}\text { Maceration } \\
\text { (topical) }\end{array}$ & Mange & he & $e x^{4}$ & 1 \\
\hline \multicolumn{8}{|l|}{ ASTERACEAE } \\
\hline Achillea millefolium L. & $\begin{array}{l}\text { Mil folhas; } \\
\text { novalgina }\end{array}$ & If & Infusion & Analgesic; fever & he & $e x^{1}$ & 3 \\
\hline Ageratum conyzoides L. & $\begin{array}{l}\text { Mentraste; } \\
\text { mentrastro }\end{array}$ & If & Infusion & $\begin{array}{c}\text { Stomach } \\
\text { discomfort; colic }\end{array}$ & he & $\mathrm{na}^{1}$ & 2 \\
\hline Artemisia absinthium L. & Losna; losnão & lf & Decoction & $\begin{array}{c}\text { Cholesterol } \\
\text { control; diabetes; } \\
\text { stomach } \\
\text { discomfort }\end{array}$ & sub & $e x^{1}$ & 2 \\
\hline Artemisia vulgaris L. & $\begin{array}{l}\text { Macaé; macaé } \\
\text { paulista }\end{array}$ & If & Infusion & $\begin{array}{c}\text { Stomach } \\
\text { discomfort; } \\
\text { abdominal pain }\end{array}$ & he & $e x^{1}$ & 2 \\
\hline
\end{tabular}


TABLE I - cont.

\begin{tabular}{|c|c|c|c|c|c|c|c|}
\hline Family/Species & Common name & $\begin{array}{c}\text { Used } \\
\text { part }\end{array}$ & $\begin{array}{l}\text { Method of } \\
\text { preparation }\end{array}$ & Mentioned use & Habit & $\begin{array}{c}\text { Status } \\
\text { of origin } \\
\text { in South } \\
\text { America* }\end{array}$ & $\begin{array}{l}\text { Number of times } \\
\text { mentioned by } \\
\text { informants }\end{array}$ \\
\hline \multicolumn{8}{|l|}{ ASTERACEAE } \\
\hline Baccharis trimera (Less.) DC. & Carqueja & lf & Infusion & Diabetes & sub & $\mathrm{na}^{1}$ & 1 \\
\hline Bidens pilosa $\mathrm{L}$. & Picão & lf; rt & Infusion & $\begin{array}{c}\text { Urinary tract } \\
\text { infection; } \\
\text { hepatitis; anaemia; } \\
\text { jaundice }\end{array}$ & he & $\mathrm{na}^{1}$ & 5 \\
\hline Dendranthema $\mathrm{sp}$ & Amor-do-senhor & lf & Infusion & Headache & he & $\mathrm{ex}^{4}$ & 1 \\
\hline Emilia fosbergii Nicolson & Serralha & lf & Juice & Depurative & he & $\mathrm{ex}^{1}$ & 1 \\
\hline Lactuca sativa $\mathrm{L}$. & Alface & lf & Fresh & Insomnia & he & $\mathrm{ex}^{1}$ & 1 \\
\hline Mikania glomerata Spreng. & Guaco & lf & Infusion & Influenza & $\mathrm{cl}$ & $\mathrm{na}^{1}$ & 3 \\
\hline $\begin{array}{l}\text { Polymnia sonchifolia Poepp. } \\
\text { Endl. }\end{array}$ & Yacon & tu & Fresh & Diabetes & $\mathrm{sb}$ & $\mathrm{na}^{4}$ & 2 \\
\hline Solidago chilensis Meyen & Arnica; ponta livre & lf & $\begin{array}{c}\text { Maceration } \\
\text { (topical) }\end{array}$ & $\begin{array}{l}\text { Wounds; pain; } \\
\text { cold } \\
\end{array}$ & sub & $\mathrm{na}^{1}$ & 3 \\
\hline $\begin{array}{l}\text { Tanacetum parthenium (L.) Sch. } \\
\text { Bip. }\end{array}$ & Artemijo & lf; fl & Decoction & $\begin{array}{c}\text { Headache; } \\
\text { stomach } \\
\text { discomfort; } \\
\text { worms; abortive }\end{array}$ & he & $e x^{2}$ & 5 \\
\hline Taraxacum officinale F.H. Wigg. & Dente de leão & lf & Infusion & Weight loss & he & $\mathrm{ex}^{1}$ & 2 \\
\hline Vernonia condensata Baker & Boldo & lf & Infusion & Liver; hangover & $\mathrm{sb}$ & $\mathrm{ex}^{1}$ & 1 \\
\hline Vernonia polyanthes Less & $\begin{array}{c}\text { Cambarazinho; assa } \\
\text { peixe; cambará }\end{array}$ & lf & Juice (topical) & Healing & $\mathrm{sb}$ & $\mathrm{na}^{1}$ & 2 \\
\hline \multicolumn{8}{|l|}{ BALSAMINACEAE } \\
\hline Impatiens balsamina $\mathrm{L}$. & Beijo branco & $\mathrm{fl}$ & Infusion & Ovary & he & $\mathrm{ex}^{2}$ & 1 \\
\hline \multicolumn{8}{|l|}{ BIXACEAE } \\
\hline Bixa orellana L. & Urucum & $\mathrm{sd}$ & Decoction & Measles & $\mathrm{sb}$ & $\mathrm{na}^{1}$ & 1 \\
\hline \multicolumn{8}{|l|}{ BORAGINACEAE } \\
\hline Symphytum officinale L. & Confrei & lf & Poultice & Healing & he & $\mathrm{ex}^{1}$ & 3 \\
\hline \multicolumn{8}{|l|}{ BRASSICACEAE } \\
\hline Brassica rapa $\mathrm{L}$. & Mostarda & lf & Decoction & Tonic & he & $\mathrm{ex}^{1}$ & 1 \\
\hline Brassica sp1 & Couve de cristo & lf & Decoction & Allergy & he & $\mathrm{ex}^{4}$ & 1 \\
\hline Brassica $\mathrm{sp} 2$ & Saião & lf & Infusion & Influenza & he & $\mathrm{ex}^{4}$ & 1 \\
\hline Coronopus didymus (L.) Sm. & Mentrustro & lf & Infusion & Inflammation & he & $\mathrm{na}^{1}$ & 1 \\
\hline Nasturtium officinale R. Br. & Agrião & lf & Fresh & $\begin{array}{c}\text { Bronchitis; } \\
\text { influenza; cold; } \\
\text { chest pain }\end{array}$ & he & $e x^{1}$ & 4 \\
\hline \multicolumn{8}{|l|}{ CARICACEAE } \\
\hline Carica papaya L. & Mamão & $\mathrm{fl}$ & Infusion & Influenza & $\mathrm{sb}$ & $\mathrm{ex}^{1}$ & 2 \\
\hline \multicolumn{8}{|l|}{ CONVOLVULACEAE } \\
\hline Cuscuta racemosa Marth. & Cipó-chumbo & $\mathrm{pt}$ & Infusion & Influenza & li & $\mathrm{na}^{4}$ & 1 \\
\hline \multicolumn{8}{|l|}{ COSTACEAE } \\
\hline Costus spicatus (Jacq.) Sw. & $\begin{array}{c}\text { Cana de macaco; } \\
\text { cana do brejo; } \\
\text { caninha do brejo }\end{array}$ & lf & Decoction & Kidney stones & sub & $\mathrm{na}^{1}$ & 5 \\
\hline \multicolumn{8}{|l|}{ CRASSULACEAE } \\
\hline Kalanchoe pinnata (Lamk.) Pers. & Fortuna & lf & Infusion & Cough & he & $\mathrm{ex}^{1}$ & 1 \\
\hline Kalanchoe brasiliensis Cambess. & Barspo; saião & lf & Fresh & $\begin{array}{c}\text { Stomach } \\
\text { discomfort; ear } \\
\text { infection } \\
\end{array}$ & he & $\mathrm{na}^{1}$ & 2 \\
\hline Kalanchoe sp & Barspo & lf & Fresh & $\begin{array}{c}\text { Stomach } \\
\text { discomfort; } \\
\text { pain; leg pain; } \\
\text { pneumonia }\end{array}$ & he & $e x^{4}$ & 6 \\
\hline
\end{tabular}


TABLE I - cont.

\begin{tabular}{lccccc}
\hline Family/Species & Common name & $\begin{array}{c}\text { Used } \\
\text { part }\end{array}$ & $\begin{array}{c}\text { Method of } \\
\text { preparation }\end{array}$
\end{tabular} Mentioned use $\begin{gathered}\text { Habit } \\
\begin{array}{c}\text { Status } \\
\text { of origin Number of times } \\
\text { in South mentioned by } \\
\text { America* }\end{array}\end{gathered}$

\section{CUCURBITACEAE}

\begin{tabular}{lccccccc}
\hline Momordica charantia L. & São caetano & - & Infusion & Fever; influenza & $\mathrm{cl}$ & $\mathrm{ex}^{1}$ & 1 \\
\hline Sechium edule (Jacq.) Sw. & Chuchu & lf; td & Decoction & $\begin{array}{c}\text { High blood } \\
\text { pressure }\end{array}$ & $\mathrm{cl}^{2}$ & $\mathrm{ex}^{1}$ & 2 \\
\hline EUPHORBIACEAE & & & & & &
\end{tabular}

\section{EUPHORBIACEAE}

\begin{tabular}{|c|c|c|c|c|c|c|c|}
\hline Euphorbia hirta L. & Olho de santa luzia & lf; bra & Infusion & $\begin{array}{c}\text { Abdominal pain } \\
\text { (diarrhoea) }\end{array}$ & he & $\mathrm{na}^{4}$ & 1 \\
\hline Ricinus communis L. & Mamona & lf; sd & Oil (topical) & Healing; toothache & $\mathrm{sb}$ & $\mathrm{ex}^{1}$ & 3 \\
\hline \multicolumn{8}{|l|}{ LAMIACEAE } \\
\hline Lavandula angustifolia Mill. & Alfazema & If & Infusion & $\begin{array}{c}\text { Influenza; } \\
\text { abdominal pain }\end{array}$ & sub & $e^{1}$ & 2 \\
\hline Leonotis nepetaefolia (L.) R. Br. & Cordão de frade & lf; fl & Decoction & $\begin{array}{c}\text { Influenza; } \\
\text { diabetes; fever; } \\
\text { back pain; } \\
\text { cholesterol } \\
\text { control; diarrhoea; } \\
\text { pressure }\end{array}$ & sub & $e^{1}$ & 4 \\
\hline Leonurus sibiricus L. & Macaé & If & Infusion & $\begin{array}{c}\text { Stomach } \\
\text { discomfort; } \\
\text { abdominal pain; } \\
\text { influenza; heart; } \\
\text { blood pressure; } \\
\text { wounds }\end{array}$ & he & $e x^{1}$ & 7 \\
\hline Mentha arvensis L. & Vick & If & Infusion & Decongestant & he & $\mathrm{ex}^{1}$ & 1 \\
\hline Mentha pulegium L. & Poejo & If & Infusion & Influenza; catarrh & he & $\mathrm{ex}^{1}$ & 5 \\
\hline Mentha sp1 & $\begin{array}{c}\text { Erva-cidreira-de- } \\
\text { horta; levante }\end{array}$ & If & Infusion & $\begin{array}{c}\text { Influenza; colic; } \\
\text { seasoning }\end{array}$ & he & $\mathrm{ex}^{4}$ & 3 \\
\hline Mentha sp2 & Hortelã & If & Infusion & $\begin{array}{c}\text { Influenza; } \\
\text { vermifuge } \\
\text { (worm); infection; } \\
\text { abdominal pain; } \\
\text { seasoning }\end{array}$ & he & $e^{4}$ & 7 \\
\hline Ocimum basilicum $\mathrm{L}$. & Manjericão & If & Infusion; fresh & $\begin{array}{c}\text { Influenza; } \\
\text { blood pressure; } \\
\text { abdominal pain }\end{array}$ & sub & $e^{1}$ & 3 \\
\hline Ocimum gratissimum L. & Titoco; alfavaca & If & Infusion & Influenza & sub & $\mathrm{ex}^{1}$ & 2 \\
\hline Ocimum selloi Benth. & $\begin{array}{c}\text { Alfavaca; } \\
\text { manjericão; } \\
\text { manjericão branco }\end{array}$ & lf; fl & Infusion & $\begin{array}{l}\text { Influenza; } \\
\text { soothing; diuretic }\end{array}$ & sub & $\mathrm{na}^{1}$ & 7 \\
\hline Origanum vulgare L. & Manjerona; orégano & If & Infusion; fresh & $\begin{array}{l}\text { Influenza; gases; } \\
\text { blood pressure }\end{array}$ & he & $e^{1}$ & 5 \\
\hline Peltodon radicans Pohl. & Erva cidreira & If & Infusion & Varicose veins & he & $\mathrm{na}^{1}$ & 1 \\
\hline $\begin{array}{l}\text { Plectranthus amboinicus (Lour.) } \\
\text { Spreng. }\end{array}$ & Hortelã pimenta & If & $\begin{array}{l}\text { Infusion; } \\
\text { Inhalation }\end{array}$ & Asthma & he & $e x^{1}$ & 1 \\
\hline Plectranthus barbatus Andrews & Boldo & If & Infusion & $\begin{array}{c}\text { Depurative; } \\
\text { headache; liver; } \\
\text { hangover }\end{array}$ & sub & $e^{1}$ & 4 \\
\hline Plectranthus ornatus Codd. & Estomazil & If & Infusion & Gastritis; ulcer & he & $\mathrm{ex}^{1}$ & 1 \\
\hline Rosmarinus officinalis L. & $\begin{array}{l}\text { Alecrim; alecrim de } \\
\text { horta }\end{array}$ & lf; bra & Infusion; fresh & $\begin{array}{c}\text { High blood } \\
\text { pressure; shortness } \\
\text { of breath; colic }\end{array}$ & sub & $e^{1}$ & 8 \\
\hline
\end{tabular}


TABLE I - cont.

\begin{tabular}{|c|c|c|c|c|c|c|c|}
\hline Family/Species & Common name & $\begin{array}{l}\text { Used } \\
\text { part }\end{array}$ & $\begin{array}{l}\text { Method of } \\
\text { preparation }\end{array}$ & Mentioned use & Habit & $\begin{array}{l}\text { Status } \\
\text { of origin } \\
\text { in South } \\
\text { America* }\end{array}$ & $\begin{array}{c}\text { Number of times } \\
\text { mentioned by } \\
\text { informants }\end{array}$ \\
\hline \multicolumn{8}{|l|}{ LAMIACEAE } \\
\hline Salvia officinalis L. & $\begin{array}{l}\text { Salvinha; sálvia; } \\
\text { salvinha miúda }\end{array}$ & lf; bra & Infusion & $\begin{array}{l}\text { Headache; delayed } \\
\text { menstruation; } \\
\text { aphthae }\end{array}$ & he & $e x^{1}$ & 3 \\
\hline \multicolumn{8}{|l|}{ LAURACEAE } \\
\hline Persea americana Mill. & Abacate & lf & Decoction & Kidney problems & $\mathrm{cl}$ & $\mathrm{ex}^{1}$ & 2 \\
\hline \multicolumn{8}{|l|}{ LEGUMINOSAE } \\
\hline Cajanus cajan (L.) Millsp. & Feijão andu & lf & $\begin{array}{c}\text { Decoction } \\
\text { (topical) }\end{array}$ & Toothache & $\mathrm{sb}$ & $e^{1}$ & 1 \\
\hline Erythrina speciosa Andrews & Barbatimão & $\mathrm{ba}$ & Decoction & Depurative & $\mathrm{cl}$ & $\mathrm{na}^{4}$ & 1 \\
\hline Stylosanthes viscosa (L.) Sw. & Salsa cavalo & $\mathrm{pt}$ & Decoction & $\begin{array}{c}\text { Uterus } \\
\text { inflammation } \\
\end{array}$ & he & $n a^{4}$ & 1 \\
\hline \multicolumn{8}{|l|}{ MALVACEAE } \\
\hline $\begin{array}{l}\text { Abelmoschus esculentus (L.) } \\
\text { Moench }\end{array}$ & Quiabo & fr; rt & Decoction & $\begin{array}{c}\text { Kidneys; } \\
\text { haemorrhage }\end{array}$ & $\mathrm{sb}$ & $\mathrm{ex}^{3}$ & 2 \\
\hline Gossypium hirsutum L. & Algodão & $\begin{array}{l}\text { lf; fl; } \\
\text { oi }\end{array}$ & $\begin{array}{l}\text { Infusion; oil } \\
\text { (topical) }\end{array}$ & $\begin{array}{c}\text { Ear pain; } \\
\text { inflammation; } \\
\text { infection }\end{array}$ & $\mathrm{sb}$ & $e^{1}$ & 3 \\
\hline Malva sp & Malva & If & Infusion & $\begin{array}{c}\text { Soothing; } \\
\text { headache; blood } \\
\text { pressure }\end{array}$ & he & $e x^{4}$ & 5 \\
\hline \multicolumn{8}{|l|}{ PAPAVERACEAE } \\
\hline Fumaria officinalis L. & Fumária & lf & Decoction & Depurative; rash & he & $\mathrm{ex}^{1}$ & 2 \\
\hline \multicolumn{8}{|l|}{ PHYLLANTHACEAE } \\
\hline Phyllanthus niruri $\mathrm{L}$. & $\begin{array}{c}\text { Rebenta pedra; } \\
\text { quebra pedra }\end{array}$ & lf; bra & Infusion & Kidney stones & he & $\mathrm{na}^{1}$ & 2 \\
\hline Phyllanthus tenellus Roxb. & Quebra-pedra & If & Infusion & Kidney stones & he & $\mathrm{na}^{1}$ & 1 \\
\hline
\end{tabular}

\section{PHYTOLACCACEAE}

Petiveria alliacea $\mathrm{L}$.

PIPERACEAE

$\frac{\text { Piper umbellatum } \mathrm{L}}{\text { PiPRACEA }}$

PLANTAGINACEAE

\begin{tabular}{|c|c|c|c|c|c|c|c|}
\hline Plantago australis Lam. & $\begin{array}{l}\text { Transagem; } \\
\text { tansagem }\end{array}$ & lf & Infusion & $\begin{array}{c}\text { Influenza; sore } \\
\text { throat; } \\
\text { smoking cessation }\end{array}$ & he & $e^{1}$ & 3 \\
\hline Plantago major L. & $\begin{array}{l}\text { Transagem; } \\
\text { tansagem }\end{array}$ & lf & Infusion & $\begin{array}{c}\text { Sore throat; } \\
\text { depurative; } \\
\text { smoking cessation }\end{array}$ & he & $e x^{1}$ & 5 \\
\hline Scoparia dulcis L. & Vassourinha doce & bra; rt & Decoction & Fever & he & $\mathrm{na}^{1}$ & 1 \\
\hline \multicolumn{8}{|l|}{ POACEAE } \\
\hline Coix lacryma-jobi L. & $\begin{array}{l}\text { Lágrima de nossa } \\
\text { senhora }\end{array}$ & $\begin{array}{l}\text { lf; rt; } \\
\text { sd }\end{array}$ & Decoction & $\begin{array}{c}\text { Fever; urine } \\
\text { problem; nutrient }\end{array}$ & he & $\mathrm{ex}^{1}$ & 3 \\
\hline $\begin{array}{l}\text { Cymbopogon citratus (DC) } \\
\text { Stapf. }\end{array}$ & $\begin{array}{l}\text { Capim cidreira; erva } \\
\text { cidreira de capim }\end{array}$ & lf & Infusion & $\begin{array}{c}\text { Soothing; } \\
\text { influenza; blood } \\
\text { pressure }\end{array}$ & he & $e x^{1}$ & 7 \\
\hline \multicolumn{8}{|l|}{ POLYGALACEAE } \\
\hline Polygala paniculata $\mathrm{L}$. & $\begin{array}{l}\text { Vassourinha de são } \\
\text { pedro }\end{array}$ & bra & Bath & Pain & he & $\mathrm{na}^{1}$ & 1 \\
\hline
\end{tabular}


TABLE I - cont.

\begin{tabular}{|c|c|c|c|c|c|c|c|}
\hline Family/Species & Common name & $\begin{array}{c}\text { Used } \\
\text { part }\end{array}$ & $\begin{array}{l}\text { Method of } \\
\text { preparation }\end{array}$ & Mentioned use & Habit & $\begin{array}{l}\text { Status } \\
\text { of origin } \\
\text { in South } \\
\text { America* }\end{array}$ & $\begin{array}{l}\text { Number of times } \\
\text { mentioned by } \\
\text { informants }\end{array}$ \\
\hline
\end{tabular}

\section{POLYGONACEAE}

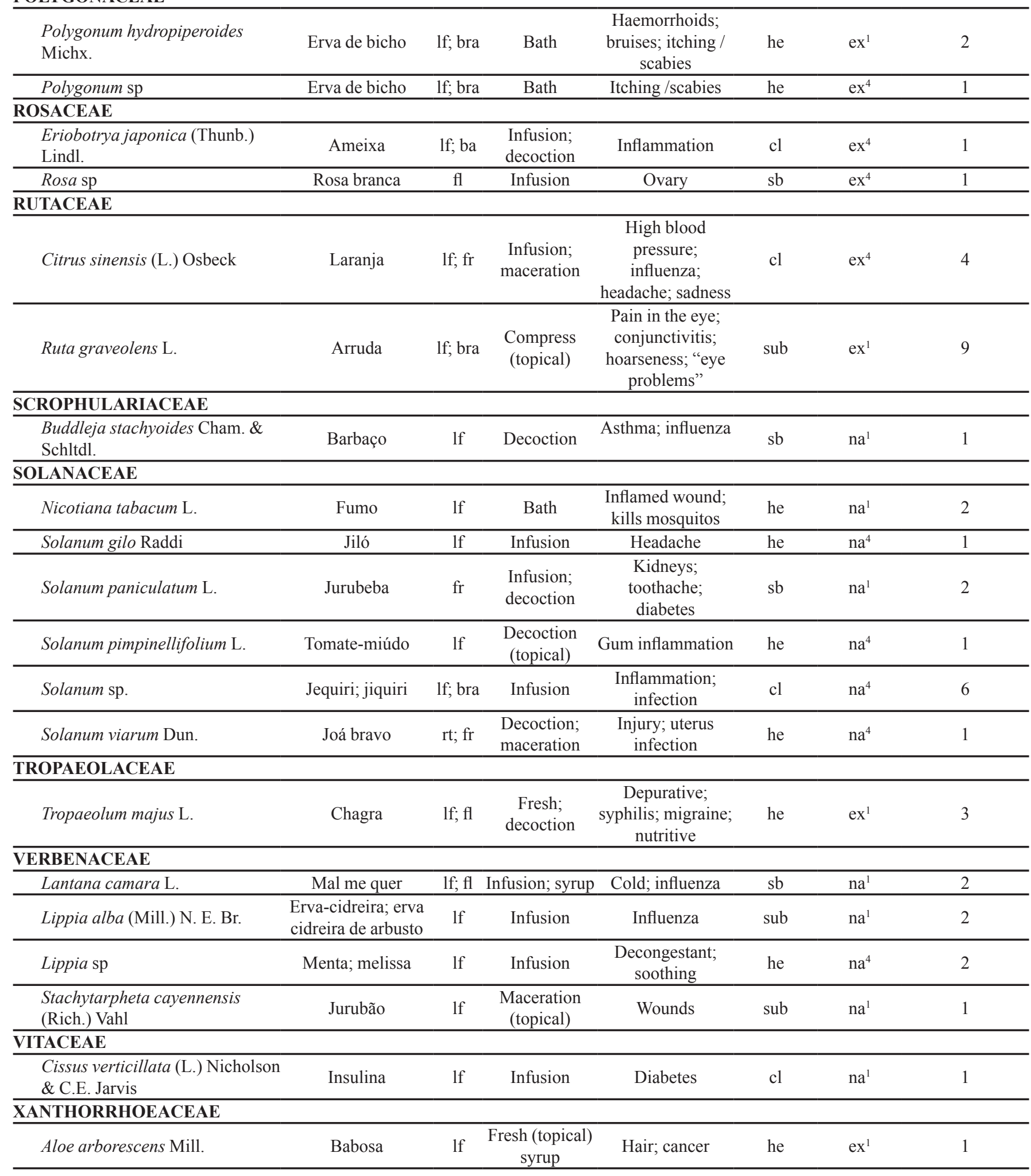


TABLE I - cont.

\begin{tabular}{|c|c|c|c|c|c|c|c|}
\hline Family/Species & Common name & $\begin{array}{c}\text { Used } \\
\text { part }\end{array}$ & $\begin{array}{l}\text { Method of } \\
\text { preparation }\end{array}$ & Mentioned use & Habit & $\begin{array}{l}\text { Status } \\
\text { of origin } \\
\text { in South } \\
\text { America* }\end{array}$ & $\begin{array}{c}\text { Number of times } \\
\text { mentioned by } \\
\text { informants }\end{array}$ \\
\hline Aloe sp & Babosa & If & $\begin{array}{c}\text { Fresh (topical) } \\
\text { syrup }\end{array}$ & $\begin{array}{l}\text { Hair; dandruff; } \\
\text { cancer; wounds; } \\
\text { abdominal pain }\end{array}$ & he & $\mathrm{ex}^{1}$ & 3 \\
\hline $\begin{array}{l}\text { Alpinia zerumbet (Pers.) B. L. } \\
\text { Burtt \& R. M. Sm. }\end{array}$ & Colônia & lf & Infusion & Blood pressure & he & $e x^{1}$ & 1 \\
\hline Zingiber officinale Roscoe & Gengibre & ri & Decoction & Influenza & he & $\mathrm{ex}^{1}$ & 1 \\
\hline
\end{tabular}

Part Used: lf=leaf, $\mathrm{fl}=$ =flower, ba=bark, bra=branches, tu=tuber, $\mathrm{rt}=$ root, $\mathrm{sd}=$ =seed, fi=filament, $\mathrm{td}=$ tendril, $\mathrm{fr}=$ fruit, $\mathrm{pt}=$ whole plant, oi $=$ oil, ri=rhizome. Habit: $\operatorname{tr}=$ tree, $s b=$ shrub, sub=subshrub, he=herbaceous, climb=climber, li=liana. Status of origin in South America: ex=exotic, na=native.

* Status of species: 1=Lorenzi and Matos, 2008, 2=Lorenzi and Souza, 2008; 3=Simpson and Ogorzaly, 2001; 4=Souza and Lorenzi, 2009.

$(6 \%)$, climber (4\%) and liana (1\%). Sixty per cent were exotic, and $40 \%$ were native to South America. Exotic species dominate other species because the study was conducted with species grown in backyards of an urban area, and medicinal plants native to Europe, Asia and Africa became popular in South America with the cultural miscegenation that historically occurred in the study area.

Different plant parts were used as medicines for the treatment of diseases by the local traditional healers. The most frequently mentioned parts were leaves $(85.0 \%)$, followed by branches (10.3\%), flowers (9.4\%), roots $(4.7 \%)$, fruits $(3.7 \%)$ and seeds, bark and tubers $(2.8 \%$ each). Leaves are traditionally the most used part for folk medical treatment, most likely because of the ease of obtaining them and because they are present on the plant throughout the majority of year, compared with flowers and fruits (Alves et al., 2008).

Forty-two of the 107 species (39\%) surveyed in this study are reported by the scientific literature, with information about the pharmacological effects of extracts and / or isolated compounds from these plants assigning the medicinal effects reported by the knowledgeable informants. The survey of pre-clinical and clinical scientific studies gave support to the therapeutic efficacies of the folk medicines reported, and this support is crucial when selecting medicinal plants for phytotherapy programs.

Interestingly, all of the informants reported that they were aware of the contraindications of herbal infusions during pregnancy. However, regarding the general perception of adverse effects with medicinal plants, only two informants $(18 \%)$ reported occurrences in certain situations. The majority of the informants attributed lack of toxicity to herbal infusions. These results show that in the sphere of public health, there is a need for more information on the rational use of medicinal plants among the population.

\section{Relative importance of species}

Four species showed CMUc values above $50 \%$ and were mentioned by four or more informants: Costus spicatus (cana de macaco/spiked spiral flag), which is used to treat kidney stones; M. pulegium (poejo/pennyroyal), which is used in the treatment of influenza; Rosmarinus officinalis (alecrim/rosemary), which is used for problems with the circulatory system; and Ruta graveolens (arruda/ rue), which is used to treat eye problems (Table II). Aside from Costus spicatus, the other species are exotic, and all four have preclinical scientific studies that support their use as mentioned by the knowledgeable informants. Table II shows CMUc indices for the most frequently mentioned species in the study.

Species showing high CMUc values may have greater potentials for medical use (Silva, Proença, 2008). This index, additionally with the survey of scientific study, represents a strategy for selecting the species to be used in local phytotherapy programs (Pinto, Amorozo, Furlan, 2006). We selected a list of plants compiled from the CMUc index (equal to or above $20 \%$ ) based on information from the scientific literature attesting to the main use mentioned by informants (Table III).

In this ethnopharmacological survey, we found two species of the genus Mentha that have not been completely identified. Mentha sp2 showed a high CMUc index for worm treatment. The ease of interspecific hybridisation within the genus Mentha contributes to the difficulty of identifying this species (Dimech et al., 2006). Mentha 
TABLE II - Surveyed species with Consensus of Main Use corrected (CMUc) percentages above 20\%

\begin{tabular}{|c|c|c|c|c|c|c|}
\hline Scientific name & Main use & NIMSU & NIMMU & $\mathrm{CMU}$ & $\mathrm{CF}$ & $\mathrm{CMUc}$ \\
\hline Alternanthera brasiliana & Antibiotic; toothache & 4 & 2 & 50.0 & 0.44 & 22.0 \\
\hline Foeniculum vulgare & Influenza; soothing & 5 & 3 & 60.0 & 0.56 & 33.0 \\
\hline Bidens pilosa & urinary infection; ovary & 5 & 2 & 40.0 & 0.56 & 22.0 \\
\hline Tanacetum parthenium & headache & 5 & 2 & 40.0 & 0.56 & 22.0 \\
\hline Nasturtium officinale & $\begin{array}{l}\text { Bronchitis; influenza, colds; chest } \\
\text { pain }\end{array}$ & 4 & 4 & 100 & 0.44 & 44.0 \\
\hline Costus spicatus & kidney stones & 5 & 5 & 100 & 0.56 & 56.0 \\
\hline Kalanchoe sp & upset stomach & 6 & 3 & 50.0 & 0.67 & 33.0 \\
\hline Leonotis nepetaefolia & Influenza; diabetes & 4 & 2 & 50.0 & 0.44 & 22.0 \\
\hline Leonurus sibiricus & stomach disorders; abdominal pain & 7 & 4 & 57.1 & 0.78 & 44.0 \\
\hline Mentha pulegium & influenza & 5 & 5 & 100 & 0.56 & 55.0 \\
\hline Mentha sp2 & Influenza; vermifuge (worms) & 7 & 3 & 42.9 & 0.78 & 33.0 \\
\hline Ocimum selloi & influenza & 7 & 3 & 42.9 & 0.78 & 33.0 \\
\hline Origanum vulgare & influenza & 5 & 3 & 60.0 & 0.56 & 33.0 \\
\hline Rosmarinus officinalis & high blood pressure; heart trouble & 8 & 5 & 62.5 & 0.89 & 55.6 \\
\hline Malva sp & soothing & 5 & 2 & 40.0 & 0.56 & 22.0 \\
\hline Plantago major & sore throat; infection of the throat & 5 & 4 & 80.0 & 0.56 & 44.0 \\
\hline Cymbopogon citratus & soothing & 7 & 4 & 57.1 & 0.78 & 44.0 \\
\hline Citrus sinensis & high blood pressure; influenza & 4 & 2 & 50.0 & 0.44 & 22.0 \\
\hline Ruta graveolens & pain in the eye; conjunctivitis & 9 & 5 & 55.6 & 1.0 & 55.6 \\
\hline Solanum sp & Inflammation; infection & 6 & 4 & 66.7 & 0.67 & 44.0 \\
\hline
\end{tabular}

NIMSU=total number of informants that mentioned the use of the species; NIMMU=number of informants that mentioned the main use of the species; $\mathrm{CMU}=$ consensus of the main use; $\mathrm{CF}=$ correction factor; $\mathrm{CMUc}=$ consensus of main use corrected.

TABLE III - Medicinal plants selected for inclusion in a local phytotherapy program on the basis of the CMUc index and scientific evidence for both safety and efficacy

\begin{tabular}{llccl}
\hline Therapeutic category & Species & PU & CMUc & Scientific evidence \\
\hline Antiparasitic & Mentha crispa & If & 33.0 & Pianowski (2000) \\
Anxiolytic & Cymbopogon citratus & If & 44.0 & Silva et al. 2010 \\
Antimicrobial & Bidens pilosa & If; rt & 22.0 & Deba et al., 2008 \\
Antispasmodic & Foeniculum vulgare & If & 33.0 & Boskabady, Khatami, Nazari (2004) \\
Anti-inflammatory & Plantago major & If & 44.0 & Guillén et al. (1997) \\
Antilipidemic & Rosmarinus officinalis & lf; bra & 55.6 & Bakirel et al. (2008) \\
Antiulcerogenic & Kalanchoe pinnata & If & 33.0 & Pal, Chaudhuri (1991) \\
Bronchodilator & Mentha pulegium & If & 55.0 & Alonso (2008) \\
Diuretic & Rosmarinus officinalis & lf; bra & 55.6 & Haloui et al. (2000) \\
\hline
\end{tabular}

$\mathrm{PU}=$ part used: $\mathrm{lf}=$ leaf, bra $=$ branch, $\mathrm{rt}=$ root

crispa, family Lamiaceae, is a hybrid derived from the cross of Mentha spicata L. and Mentha suaveolens Ehlh (Matos, 1991). Additionally, M. crispa is known as Mentha $x$ villosa Hudson or Mentha spicata L.

A similar case is observed with species of the genus Kalanchoe. In Brazil, the two most frequent species are $K$. brasiliensis Camb. and K. pinnata (Lamk.) Pers., both of which are known as "folha-da-fortuna" or "saião". In this study, we identified the species $K$. brasiliensis. The other species was not completely identified because the plants did not have flowers at the time of collection; however, as the plants were used to treat stomach discomfort, they were 
likely of the $K$. pinnata species, which has scientific evidence of antiulcer activity (Amaral, Simões, Ferreira, 2005).

Based on these above cases, it was vital to define the species correctly based on the studies regarding therapeutic efficacy because that information would allow for their inclusion in phytotherapy programs and would ensure only cultivated plants from certified nursery sources are used in the future. In this study, the species M. crispa and $K$. pinnata were verified for use in the phytotherapy program. Furthermore, these and the other species selected for the phytotherapy program showed CMUc indices above $22 \%$ and provided scientific evidence for both safety and efficacy, meeting the medical criteria for prescribing phytotherapeutics (Ribeiro, Leite, Barros, 2005).

\section{CONCLUSION}

The ethnopharmacological survey of medicinal plants conducted with knowledgeable informants in a community provided information on the medicinal properties of 107 species of plants used in local traditional medicine. To implement a local program utilising the herbal medicines, methodology implementing the CMUc index, combined with a scientific information survey, was used to compile a list of medicinal plants for use in nine different therapeutic classes, which resulted from eight selected species. The species selected were Mentha crispa (antiparasitic), Cymbopogon citratus (anxiolytic), Bidens pilosa (antimicrobial), Foeniculum vulgare (antispasmodic), Plantago major (anti-inflammatory), Rosmarinus officinalis (antilipidemic and diuretic), Kalanchoe pinnata (antiulcerogenic) and Mentha pulegium (bronchodilator).

The combined use of quantitative data from the ethnopharmacological survey and the scientific literature to select medicinal plants for phytotherapy programs benefited from the use of information from local residents and the scientific community. This approach seeks to enhance the involvement of the community and health professionals in the local phytotherapy program.

\section{ACKNOWLEDGMENTS}

We are grateful to the residents of the community of Nova Viçosa for their cooperation during the interviews.

\section{REFERENCES}

ALBUQUERQUE, U.P.; LUCENA, R.F.P. Seleção e escolha dos informantes. In: ALBUQUERQUE, U.P.; LUCENA, R.F.P. (Orgs.). Métodos e técnicas na pesquisa etnobotânica. Recife: Livro Rápido/NUPEEA, 2004. 189 p.
ALONSO, J. Tratado de fitofármacos e nutracéuticos. Buenos Aires: Corpus, 2008. 130 p.

ALVES, E.O.; MOTA, J.H.; SOARES, T.S.; VIEIRA, M.C.; SILVA, C.B. Levantamento etnobotânico e caracterização de plantas medicinais em fragmentos florestais de Dourados-MS. Ciênc. Agrotec., v.32, n.2, p.651-658, 2008.

AMARAL, A.C.F.; SIMÕES, E.V.; FERREIRA, J.L.P. Coletânea científica de plantas de uso medicinal. FIOCRUZ. Rio de Janeiro: Abifito, 2005. 222 p.

AMARAL, C.N.; GUARIM-NETO, G. Os quintais como espaços de conservação e cultivo de alimentos: um estudo na cidade de Rosário Oeste (Mato Grosso, Brasil). Ciênc. Hum., v.3, n.3, p.329-341, 2008.

AMOROZO, M.C.M.; GÉLY, A. Uso de plantas medicinais por caboclos do Baixo Amazonas, Barcarena, PA, Brasil. Bol. Mus. Para. Emílio Goeldi sér. Bot., v.4, n.1, p.47-131, 1988.

ANGIOSPERM PHYLOGENY GROUP. An update of the Angiosperm Phylogeny Group classification for the orders and families of flowering plants: APG III. Bot. J. Linn. Soc., v.161, n.2, p.105-121, 2009.

ARATO, H.D.; MARTINS, S.V.; FERRARI, S.H.S. Produção e decomposição de serapilheira em um sistema agroflorestal implantado para recuperação de área degradada em ViçosaMG. Rev. Árvore, v.27, n.5, p.715-721, 2003.

BAKIREL, T.; BAKIREL, U.; KELEŞ, O.U.; ÜLGEN, S.G.; YARDIBI, H. In vivo assessment of antidiabetic and antioxidant activities of rosemary (Rosmarinus officinalis) in alloxan-diabetic rabbits. J. Ethnopharmacol., v.116, n.1, p.64-73, 2008.

BERMÚDEZ, A.; OLIVEIRA-MIRANDA, M.A.; VELÁZQUEZ, D. La investigación etnobotánica sobre plantas medicinales: Una revisión de sus objetivos y enfoques actuales. Interciencia, v.30, n.8, p.453-459, 2005.

BORÉM, R.A.T.; OLIVEIRA-FILHO, A.T. Fitossociologia do estrato arbóreo em uma toposseqüência alterada de mata atlântica, no município de Silva Jardim-RJ, Brasil. Rev. Árvore, v.26, n.6, p.727-742, 2002.

BOSKABADY, M.H.; KHATAMI, A.; NAZARI, A. Possible mechanism(s) for relaxant effects of Foeniculum vulgare on guinea pig tracheal chains. Pharmazie, v.59, n.7, p.561564, 2004. 
BRASIL. Medida provisória n.2.186-16, 23 Aug. 2001. Dispõe sobre o acesso ao patrimônio genético, a proteção e o acesso ao conhecimento tradicional associado, a repartição de benefícios e o acesso à tecnologia e transferência de tecnologia para sua conservação e utilização, e dá outras providências. Available at: <www.planalto.gov.br/ ccivil_03/mpv/2186-16.htm>. Accessed on: 02 Apr. 2012.

BRASIL. Ministério da Saúde. Secretaria de Atenção à Saúde. Departamento de Atenção Básica. Política Nacional de Práticas Integrativas e Complementares no SUS - PNPICSUS. Brasília: Ministério da Saúde, 2006. 92 p.

BRASIL. Ministério da Saúde. Secretaria de Ciência, Tecnologia e Insumos Estratégicos. Departamento de Assistência Farmacêutica e Insumos Estratégicos. Programa Nacional de Plantas Medicinais e Fitoterápicos. Brasília: Ministério da Saúde, 2009. 136 p.

BRASIL. Agência Nacional de Vigilância Sanitária - Anvisa. Instrução Normativa n.5, 31 Mar. 2010. Estabelece a Lista de referências bibliográficas para avaliação de segurança e eficácia de medicamentos fitoterápicos. Diário Oficial da União, Brasília, 05 Apr. 2010. Seção 1, p.91.

BRIDSON, D.; FORMAN, L. The herbarium: handbook. 3ed. Kew: Royal Botanic Gardens, 1999. 334 p.

BRITO, M.A.; COELHO, M.F. Os quintais agroflorestais em regiões tropicais - unidades auto-sustentáveis. Rev. Bot. App. Agric. Trop., v.4, n.1, p.7-35, 2000.

BRUHN, J.G.; HOLMSTEDT, B. Ethnopharmacology: objectives, principles and perspectives. In: BEAL, J.L.; REINHARD, E. (Eds.). Natural products as medicinal agents. Struttgart: Hippokrates Verlag, 1981. p.405-430.

BRUMMIT, R.K.; POWELL, C.E. Authors of plant names. Kew: Royal Botanic Gardens, 1992. 732 p.

CAMEJO-RODRIGUES, J.S. Contributo para o estudo etnobotânico das plantas medicinais e aromáticas no Parque Natural da Serra de S. Mamede. Lisboa, 2001. 249 p. [Relatório de Estágio de Licenciatura em Biologia pela Faculdade de Ciências da Universidade de Lisboa].

CARVALHO, A.F.; COSTA, L.C.; NOVAES, D.M.; PINTO, M.P.A.; AROUCA, N.E. Agricultura urbana: alternativa de segurança alimentar e geração de renda, Viçosa, MG. In: Congresso brasileiro de extensão universitária, 2., Belo Horizonte, 2004. Anais. Belo Horizonte: UFMG, 2004. p.7.
DEBA, F.; XUAN, T.D.; YASUDA, M.; TAWATA, S. Chemical composition and antioxidant, antibacterial and antifungal activities of the essential oils from Bidens pilosa Linn. var. Radiata. Food Control, v.19, n.4, p.346-352, 2008.

DIEGUES, A.C.; ARRUDA, R.S.V.; SILVA, V.C.F.; FIGOLS, F.A.B.; ANDRADE, D. Os saberes tradicionais e a biodiversidade no Brasil. São Paulo: MMA/ NUPAUB, 2000. 189 p.

DIMECH, G.S.; GONÇALVES, E.S.; ARAÚJO, A.V.; ARRUDA, V.M.; BARATELLA-EVÊNCIO, L.; WANDERLEY, A.G. Evaluation of the hydroalcoholic extract of Mentha crispa on the reproductive performance in Wistar rats. Braz. J. Pharmacog., v.16, n.2, p.152-157, 2006.

ELISABETSKY, E. Etnofarmacologia. Cienc. Cult., v.55, n.3, p.35-36, 2003.

FUNDAÇÃO JOÃO PINHEIRO. Perfil demográfico do Estado de Minas Gerais - 2002. Belo Horizonte: Centro de Estatística e Informações, 2003. 111 p.

GUILLÉN, M.E.N.; EMIM, J.A.S.; SOUCCAR, C.; LAPA, A.J. Analgesic and anti-inflammatory activities of the aqueous extract of Plantago major L. Pharm. Biol., v.35, n.2, p.99104, 1997.

HALOUI, M.; LOUEDEC, L.; MICHEL, J.B.; LYOUSSI, B. Experimental diuretic effects of Rosmarinus officinalis and Centaurium erythraea. J. Ethnopharmacol., v.71, n.3, p.465-472, 2000.

INSTITUTO BRASILEIRO DE GEOGRAFIA E ESTATÍSTICA. Censo Demográfico 2010, 2010.

INTERNATIONALSOCIETYOFETHNOPHARMACOLOGY, 2012. ISE Constitution. Available at: <http://www. ethnopharmacology.org/ISE_constitution.htm $>$. Accessed on: 28 Mar. 2012.

THE INTERNATIONAL PLANT NAMES INDEX, 2009. Available at: $<$ http://www.ipni.org $>$. Accessed on: 15 Jun. 2009.

LEWIS, G.; SCHRINE, B.; MACKINDER, B.; LOCK, M. Legumes of the World. Kew: Royal Botanic Gardens, 2005. $577 \mathrm{p}$. 
LORENZI, H.; MATOS, F.J.A. Plantas medicinais no Brasil: nativas e exóticas. 2ed. Nova Odessa: Instituto Plantarum, 2008.544 p.

LORENZI, H.; SOUZA, H.M. Plantas ornamentais no Brasil: arbustivas, herbáceas e trepadeiras. 4.ed. Nova Odessa: Instituto Plantarum, 2008. 1120 p.

MARQUES, M.B. Patentes farmacêuticas e acessibilidade aos medicamentos no Brasil. Hist. Ciênc. Saúde-Manguinhos, v.7, n.1, p.7-21, 2000.

MATOS, F.J.A. Farmácias vivas: sistema de utilização de plantas medicinais projetados para pequenas comunidades. Fortaleza: Universidade Federal do Ceará, 1991. 75 p.

MELLO, F.A.O. Análise do processo de formação da paisagem urbana do município de Viçosa. Viçosa, 2002. 103 p. [Thesis of Master's Degree. Faculty of Forestry Engineering, Federal University of Viçosa. Postgraduate Degree Programn in Forestry Science].

MING, L.C.; JUNIOR, A.A. 2005. Aspectos Etnobotânicos de Plantas Medicinais na Reserva Extrativista "Chico Mendes". Florística e Botânica Econômica do Acre, Brasil. The New York Botanical Garden. Available at: http://www. nybg.org/bsci/acre/www1/medicinal.html. Accessed on: 20 Jun. 2009.

MITTERMEIER, R.A.; MITTERMEIER, C.G.; BROOKS, T.M.; PILGRIM, J.D.; KONSTANT, W.R.; FONSECA, G.A.B.; KORMOS, C. Wilderness and biodiversity conservation. Proc. Natl. Acad. Sci., v.100, n.18, p.10309$10313,2003$.

MOURA, C.L.; ANDRADE, L.H.C. Etnobotânica em quintais urbanos nordestinos: um estudo no bairro da Muribeca, Jaboatão dos Guararapes - PE. R. Bras. Bioci., v.5, n.1, p.219-221, 2007.

MYERS, N.; MITTERMEIER, R.A.; MITTERMEIER, C.G.; FONSECA, G.A.B.; KENT, J. Biodiversity hotspots for conservation priorities. Nature, v.403, n.6772, p.853-858, 2000

PAL, S.; CHAUDHURI, A.K.N. Studies on the anti-ulcer activity of a Bryophyllum pinnatum leaf extract in experimental animals. J. Ethnopharmacol., v.33, n.1-2, p.97-102, 1991.
PAULA, A.; SILVA, A.F.; SOUZA, A.L.; SANTOS, F.A.M. Alterações florísticas ocorridas num período de quatorze anos na vegetação arbórea de uma Floresta Estacional Semidecidual em Viçosa-MG. Rev. Árvore, v.26, n.6, p.743$749,2002$.

PIANOWSKI, L.F. Desenvolvimento farmacêutico de um produto fitoterápico. Porto (Portugal), 2000. 82 p. [Thesis of PhD degree. Faculty of Pharmacy, University of Porto. $\mathrm{PhD}$ Programn in Pharmaceutical Technology].

PILLA, M.A.C.; AMOROZO, M.C.M.; FURLAN, A. Obtenção e uso das plantas medicinais no distrito de Martim Francisco, Município de Mogi-Mirim, SP, Brasil. Acta Bot. Bras., v.20, n.4, p.789-802, 2006.

PINTO, E.P.P.; AMOROZO, M.C.M.; FURLAN, A. Conhecimento popular sobre plantas medicinais em comunidades rurais de mata atlântica - Itacaré, BA, Brasil. Acta Bot. Bras., v.20, n.4, p.751-762, 2006.

PRUSKI, J.F.; SANCHO, G. Asteraceae. In: SMITH, N. (Ed.). Flowering plants of the Neotropics. Princeton: Princeton University Press, 2004. p.33-39.

RIBEIRO, A.Q.; LEITE, J.P.V.; BARROS, A.M.D. Perfil de utilização de fitoterápicos em farmácias comunitárias de Belo Horizonte sob a influência da legislação nacional. Rev. Bras. Farmacogn., v.15, n.1, p.65-70, 2005.

SANTOS, M.R.A.; LIMA, M.R.; FERREIRA, M.G. Uso de plantas medicinais pela população de Ariquemes, em Rondônia. Hortic. Bras., v.26, n.2, p.244-250, 2008.

SANTOS, S.; CORREIA, A.I.D.; FIGUEIREDO, A.C.; DIAS, L.S.; DIAS, A.S. Plantas medicinais da Península de Setúbal. Contribuição para o conhecimento da sua relevância etnobotânica. In: FIGUEIREDO, A.C.; BARROSO, J.G.; PEDRO, L.G. (Eds.). Potencialidades e aplicações das plantas aromáticas e medicinais. Curso Teórico-Prático. 3. ed. Lisboa: Faculdade de Ciências da Universidade de Lisboa, Centro de Biotecnologia Vegetal, 2007. p.175-182.

SILVA, C.S.P.; PROENÇA, C.E.B. Uso e disponibilidade de recursos medicinais no município de Ouro Verde de Goiás, GO, Brasil. Acta Bot. Bras., v.22, n.2, p.481-492, 2008. 
SILVA, M.R.; XIMENES, R.M.; COSTA, J.G.; LEAL, L.K.; LOPES, A.A.; VIANA, G.S. Comparative anticonvulsant activities of the essential oils (EOs) from Cymbopogon winterianus Jowitt and Cymbopogon citratus (DC) Stapf. in mice. Naunyn-Schmiedeberg's Arch. Pharmacol., v.381, n.5, p.415-426, 2010.

SIMPSON, B.B.; OGORZALY, M.C. Economic botany: plants in our world. 3 ed. New York: McGraw-Hill Companies, Inc., 2001. 529 p.

SOUZA, V.C.; LORENZI, H. Botânica sistemática: guia ilustrado para identificação das famílias de Angiospermas da flora brasileira, baseado em APG II. 2 ed. Nova Odessa: Instituto Plantarum, 2009. 704 p.
TABARELLI, M.; PINTO, L.P.; SILVA, J.M.C.; HIROTA, M.M.; BEDÊ, L.C. Desafios e oportunidades para a conservação da biodiversidade na Mata Atlântica brasileira. Megadiversidade, v.1, n.1, p.132-138, 2005.

VELOSO, H.P.; RANGEL-FILHO, A.L.R.; LIMA, J.C. Classificação de vegetação brasileira, adaptada a um sistema universal. Rio de Janeiro: IBGE, 1991. 124 p.

Received for publication on $10^{\text {th }}$ October 2011 Accepted for publication on $14^{\text {th }}$ May 2012 
